\title{
Front Matter: Volume 7683
}

, "Front Matter: Volume 7683," Proc. SPIE 7683, Energy Harvesting and Storage: Materials, Devices, and Applications, 768301 (28 May 2010); doi: 10.1117/12.866495

SPIE Event: SPIE Defense, Security, and Sensing, 2010, Orlando, Florida, United SPIE. States 


\section{PROCEEDINGS OF SPIE}

\section{Energy Harvesting and Storage: Materials, Devices, and Applications}

Nibir K. Dhar

Priyalal S. Wijewarnasuriya

Achyut K. Dutta

Editors

5-6 April 2010

Orlando, Florida, United States

Sponsored and Published by

SPIE

Volume 7683 
The papers included in this volume were part of the technical conference cited on the cover and title page. Papers were selected and subject to review by the editors and conference program committee. Some conference presentations may not be available for publication. The papers published in these proceedings reflect the work and thoughts of the authors and are published herein as submitted. The publisher is not responsible for the validity of the information or for any outcomes resulting from reliance thereon.

Please use the following format to cite material from this book:

Author(s), "Title of Paper," in Energy Harvesting and Storage: Materials, Devices, and Applications, edited by Nibir K. Dhar, Priyalal S. Wijewarnasuriya, Achyut K. Dutta, Proceedings of SPIE Vol. 7683 (SPIE, Bellingham, WA, 2010) Article CID Number.

ISSN 0277-786X

ISBN 9780819481474

Published by

SPIE

P.O. Box 10, Bellingham, Washington 98227-0010 USA

Telephone +1 3606763290 (Pacific Time) · Fax +1 3606471445

SPIE.org

Copyright (C) 2010, Society of Photo-Optical Instrumentation Engineers

Copying of material in this book for internal or personal use, or for the internal or personal use of specific clients, beyond the fair use provisions granted by the U.S. Copyright Law is authorized by SPIE subject to payment of copying fees. The Transactional Reporting Service base fee for this volume is $\$ 18.00$ per article (or portion thereof), which should be paid directly to the Copyright Clearance Center (CCC), 222 Rosewood Drive, Danvers, MA 01923. Payment may also be made electronically through CCC Online at copyright.com. Other copying for republication, resale, advertising or promotion, or any form of systematic or multiple reproduction of any material in this book is prohibited except with permission in writing from the publisher. The CCC fee code is 0277-786X/10/\$18.00.

Printed in the United States of America.

Publication of record for individual papers is online in the SPIE Digital Library.

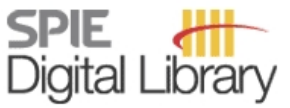

SPIEDigitalLibrary.org

Paper Numbering: Proceedings of SPIE follow an e-First publication model, with papers published first online and then in print and on CD-ROM. Papers are published as they are submitted and meet publication criteria. A unique, consistent, permanent citation identifier (CID) number is assigned to each article at the time of the first publication. Utilization of CIDs allows articles to be fully citable as soon they are published online, and connects the same identifier to all online, print, and electronic versions of the publication. SPIE uses a six-digit CID article numbering system in which:

- The first four digits correspond to the SPIE volume number.

- The last two digits indicate publication order within the volume using a Base 36 numbering system employing both numerals and letters. These two-number sets start with 00, 01, 02, 03, 04, $05,06,07,08,09,0 A, 0 B \ldots 0 Z$, followed by 10-1Z, 20-2Z, etc.

The CID number appears on each page of the manuscript. The complete citation is used on the first page, and an abbreviated version on subsequent pages. Numbers in the index correspond to the last two digits of the six-digit CID number. 


\section{Contents}

vii Conference Committee

\section{SESSION 1 ADVANCED ENERGY STORAGE TECHNOLOGIES: BATTERY AND FUEL CELLS I}

768303 Nanostructured electrode materials for Li-ion battery (Invited Paper) [7683-01]

P. Balaya, K. Saravanan, S. Hariharan, National Univ. of Singapore (Singapore)

768309 Improved layered mixed transition metal oxides for Li-ion batteries (Invited Paper) [7683-08]

M. M. Doeff, T. Conry, J. Wilcox, Lawrence Berkeley National Lab. (United States)

\section{SESSION 2 ADVANCED ENERGY STORAGE TECHNOLOGIES: BATTERY AND FUEL CELLS II}

7683 OA Diagnostic studies on lithium-ion cells at Argonne National Laboratory: an overview (Invited Paper) [7683-09]

D. P. Abraham, Argonne National Lab. (United States)

7683 OB Understanding Li-ion battery processes at the atomic- to nano-scale (Invited Paper) [7683-10]

J. P. Sullivan, J. Huang, M. J. Shaw, A. Subramanian, N. Hudak, Sandia National Labs. (United States); Y. Zhan, J. Lou, Rice Univ. (United States)

7683 OC In-house fabrication and testing capabilities for Li and Li-ion 18650 cells (Invited Paper) [7683-11]

G. Nagasubramanian, Sandia National Labs. (United States)

7683 OF Higher energy and safety of lithium-ion batteries with ionic liquid electrolyte (Invited Paper) [7683-14]

S. Komaba, N. Yabuuchi, Tokyo Univ. of Science (Japan); Y. Katayama, T. Miura, Keio Univ. (Japan)

\section{SESSION 3 ADVANCED ENERGY STORAGE TECHNOLOGIES AND APPLICATIONS}

$76830 \mathrm{~J}$ Silicates and titanates as high-energy cathode materials for Li-ion batteries (Invited Paper) [7683-19]

R. Dominko, National Institute of Chemistry (Slovenia)

7683 OK Self-regulating fiber fuel cell (Invited Paper) [7683-20]

S. J. Eickhoff, Honeywell ACS Labs. (United States) 
7683 ON Multijunction single-crystal CdTe-based solar cells: opportunities and challenges (Invited Paper) [7683-23]

S. Sivananthan, J. W. Garland, M. W. Carmody, EPIR Technologies, Inc. (United States)

$768300 \quad$ High-efficiency solar cells based on micro-nano scale structures (Invited Paper) [7683-24]

A. K. Dutta, R. Olah, G. Mizuno, R. Sengupta, J.-H. Park, Banpil Photonics, Inc. (United States); P. Wijewarnasuriya, U.S. Army Research Lab. (United States); N. K. Dhar, U.S. Army Research Lab. (United States) and DARPA Microelectronics Technology Office (United States)

$76830 Q \quad$ GaAs-based quantum well solar cells for defense applications [7683-26]

R. E. Welser, A. K. Sood, Y. R. Puri, Magnolia Optical Technologies, Inc. (United States); O. A. Laboutin, Kopin Corp. (United States); N. K. Dhar, Microelectronics Technology Office/DARPA (United States); P. S. Wijewarnasuriya, U.S. Army Research Lab. (United States)

7683 OR High-efficiency InN-based quantum dot solar cells for defense applications (Invited Paper) [7683-27]

R. E. Welser, A. K. Sood, Y. R. Puri, Magnolia Optical Technologies, Inc. (United States); O. A. Laboutin, Kopin Corp. (United States); L. J. Guido, Virginia Polytechnic Institute and State Univ. (United States); N. K. Dhar, DARPA Microelectronics Technology Office (United States); P. S. Wijewarnasuriya, U.S. Army Research Lab. (United States)

\section{SESSION 5 NOVEL THERMOELECTRIC TECHNOLOGIES}

7683 OS Multidimensional nanoscopic approaches to new thermoelectric materials (Invited Paper) [7683-28]

D. S. Dudis, J. B. Ferguson, M. Check, J. E. Schmidt, E. R. Kemp, T. Robbins, Air Force Research Lab. (United States); J. A. Shumaker, C. Chen, Univ. of Dayton Research Institute (United States); H. A. Seibel II, Universal Technology Corp. (United States)

7683 OT Thermosensitive conductivity of CdS thin film [7683-29]

X. Zeng, C. A. Kwapich, Y. X. Gan, A. H. Jayatissa, The Univ. of Toledo (United States)

\section{SESSION 6 ADVANCED THERMOELECTRIC AND NOVEL ENERGY GENERATION TECHNOLOGIES}

$7683 \mathrm{OV}$ Novel metal/semiconductor nanocomposite and superlattice materials and devices for thermoelectrics (Invited Paper) [7683-31]

J. M. O. Zide, Univ. of Delaware (United States); H. Lu, Univ. of California, Santa Barbara (United States); T. Onishi, Univ. of California, Santa Cruz (United States); J. L. Schroeder, Purdue Univ. (United States); J. E. Bowers, Univ. of California, Santa Barbara (United States); N. P. Kobayashi, Univ. of California, Santa Cruz (United States); T. D. Sands, Purdue Univ. (United States); A. C. Gossard, Univ. of California, Santa Barbara (United States); A. Shakouri, Univ. of California, Santa Cruz (United States)

7683 oW Thin-film superlattice thermoelectric materials and device technologies for energy harvesting applications (Invited Paper) [7683-32]

C. D. Stokes, RTI International (United States); E. A. Duff, QUASAR, Inc. (United States); M. J. Mantini, B. A. Grant, P. P. Barletta, R. Venkatasubramanian, RTI International (United States) 
$76830 \mathrm{X}$ Ultra-low-power conversion and management techniques for thermoelectric energy harvesting applications (Invited Paper) [7683-33]

J. W. Fleming, Luna Innovations Inc. (United States)

7683 OY Harvesting vibration energy using nonlinear oscillations of an electromagnetic inductor [7683-35]

C. Lee, D. Stamp, N. R. Kapania, J. O. Mur-Miranda, Franklin W. Olin College of Engineering (United States)

$76830 Z$ Microcombustor-thermoelectric power generator for 10-50 watt applications (Invited Paper) [7683-36]

D. S. Marshall, S. T. Cho, CUBE Technology, Inc. (United States)

POSTER SESSION

768310 Low-temperature crystallization of $\mathrm{TiO}_{2}$ films by sputter deposition [7683-37]

Y. Taga, N. Yamada, Chubu Univ. (Japan)

768311 Structural, surface, optical, and mechanical properties of $\mathrm{Zn}_{3} \mathrm{~N}_{2}$ thin films prepared by sputtering deposition [7683-38]

T. Wen, N. Jiang, D. G. Georgiev, A. H. Jayatissa, The Univ. of Toledo (United States)

768312 Purely sonochemical route for oriented zinc oxide nanowire growth on arbitrary substrate [7683-39]

A. P. Nayak, A. M. Katzenmeyer, J.-Y. Kim, M. K. Kwon, Univ. of California, Davis (United States); Y. Gosho, Yamatake Corp. (Japan) and BSAC Berkley (United States); M. S. Islam, Univ. of California, Davis (United States)

768313 Transfer of micro/nano-scale pillars and wires on conducting thermoplastic composite coated arbitrary substrates [7683-40]

Jayaraman, L. V., M. Ombaba, A. M. Katzenmeyer, M. S. Islam, Univ. of California, Davis (United States)

768314 Electrical optimization of power delivery through thick steel barriers using piezoelectric transducers [7683-41]

T. J. Lawry, K. R. Wilt, S. Roa-Prada, J. D. Ashdown, G. J. Saulnier, H. A. Scarton, Rensselaer Polytechnic Institute (United States); P. K. Das, Univ. of California, San Diego (United States);

J. D. Pinezich, Advanced Acoustic Concepts, Inc. (United States)

768318 Performance enhancements of dye sensitized nanostructured solar cells using PbS quantum dots [7683-45]

P. K. D. D. P. Pitigala, K. Tennakone, A. G. U. Perera, Georgia State Univ. (United States)

7683 1B High-efficiency integrated piezoelectric energy harvesting systems [7683-49]

A. Hande, P. Shah, Texas MicroPower, Inc. (United States)

Author Index 
Downloaded From: https://www.spiedigitallibrary.org/conference-proceedings-of-spie on 26 Apr 2023

Terms of Use: https://www.spiedigitallibrary.org/terms-of-use 


\title{
Conference Committee
}

\author{
Symposium Chair
}

Michael T. Eismann, Air Force Research Laboratory (United States)

Symposium Cochair

William Jeffrey, HRL Laboratories, LLC (United States)

Conference Chairs

Nibir K. Dhar, DARPA Microelectronics Technology Office (United States)

Priyalal S. Wijewarnasuriya, U.S. Army Research Laboratory (United States)

Achyut K. Dutta, Banpil Photonics, Inc. (United States)

Program Committee

Pulickel M. Ajayan, Rice University (United States)

Palani Balaya, National University of Singapore (Singapore)

Fow-Sen Choa, University of Maryland, Baltimore County (United States)

M. Saif Islam, University of California, Davis (United States)

Ahalapitiya Hewage Jayatissa, University of Toledo (United States)

Nobuhiko Paul Kobayashi, University of California, Santa Cruz (United States)

Pat McGrath, Booz Allen Hamilton (United States)

Authi A. Narayanan, The Boeing Company (United States)

Robert Olah, Banpil Photonics, Inc. (United States)

Unil A. Perera, Georgia State University (United States)

A. Fred Semendy, Army Research Laboratory (United States)

Rao Surampudi, Jet Propulsion Laboratory (United States)

Patrick Taylor, Army Research Laboratory (United States)

Sudhir B. Trivedi, Brimrose Corporation of America (United States)

Rama Venkatasubramanian, RTI International (United States)

\section{Session Chairs}

1 Advanced Energy Storage Technologies: Battery and Fuel Cells I

Nibir K. Dhar, Microelectronics Technology Office/DARPA (United States) 
2 Advanced Energy Storage Technologies: Battery and Fuel Cells II Deryn Chu, U.S. Army Research Laboratory (United States)

3 Advanced Energy Storage Technologies and Applications Nibir K. Dhar, DARPA Microelectronics Technology Office (United States)

Achyut K. Dutta, Banpil Photonics, Inc. (United States)

$4 \quad$ Advanced Photovoltaic Cell Technologies

Priyalal S. Wijewarnasuriya, U.S. Army Research Laboratory (United States)

Authi A. Narayanan, The Boeing Company (United States)

$5 \quad$ Novel Thermoelectric Technologies

Rama Venkatasubramanian, RTI International (United States)

Patrick Taylor, U.S. Army Research Laboratory (United States)

6 Advanced Thermoelectric and Novel Energy Generation Technologies

Patrick Taylor, U.S. Army Research Laboratory (United States)

Rama Venkatasubramanian, RTI International (United States) 